\title{
3,4-Dihydroxyphenylacetaldehyde synthase and cuticle formation in insects
}

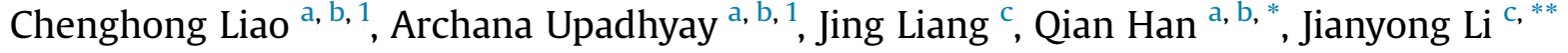 \\ ${ }^{a}$ Key Laboratory of Tropical Biological Resources of Ministry of Education, Hainan University, Haikou, Hainan 570228, China \\ ${ }^{\mathrm{b}}$ Laboratory of Tropical Veterinary Medicine and Vector Biology, Hainan Key Laboratory of Sustainable Utilization of Tropical Bioresources, Institute of \\ Tropical Agriculture and Forestry, Hainan University, Haikou, Hainan 570228, China \\ ${ }^{\mathrm{c}}$ Department of Biochemistry, Virginia Tech, Blacksburg, VA 24061, USA
}

\section{A R T I C L E I N F O}

\section{Article history:}

Received 12 September 2017

Received in revised form

28 October 2017

Accepted 13 November 2017

Available online 15 November 2017

\section{Keywords:}

3,4-Dihydroxyphenylacetaldehyde

Insect

Cuticle

$\alpha$-methyl dopa resistant protein

3,4-Dihydroxyphenylacetaldehyde synthase

\begin{abstract}
A B S T R A C T
Cuticle is the most important structure that protects mosquitoes and other insect species from adverse environmental conditions and infections of microorganism. The physiology and biochemistry of insect cuticle formation have been studied for many years and our understanding of cuticle formation and hardening has increased considerably. This is especially true for flexible cuticle. The recent discovery of a novel enzyme that catalyzes the production of 3,4-dihydroxyphenylacetaldehyde (DOPAL) in insects provides intriguing insights concerning the flexible cuticle formation in insects. For convenience, the enzyme that catalyzes the production DOPAL from L-dopa is named DOPAL synthase. In this mini-review, we summarize the biochemical pathways of cuticle formation and hardening in general and discuss DOPAL synthase-mediated protein crosslinking in insect flexible cuticle in particular.
\end{abstract}

(c) 2017 Elsevier Ltd. All rights reserved.

\section{Introduction}

In insects, the cuticle provides protection against physical injury and flexibility in inter-segmental and joint areas for mobility. The cuticle covers the body of the insect and acts as an effective barrier between the insect and its surroundings. It provides protection against desiccation, microorganisms, and predators, and simultaneously acting as an exoskeleton providing the attachment sites for muscles (Gibbs et al., 1991; Moussian, 2010; Ortiz-Urquiza and Keyhani, 2013). Our understanding of cuticular structures has largely been derived from studies of Diptera and Lepidoptera species (such as Drosophila melanogaster, Manduca sexta, etc.) (Rebers and Riddiford, 1988; Suderman et al., 2003; Moussian et al., 2006; Shibata et al., 2010; Pesch et al., 2017). Based on physical characteristics, cuticle can be further classified into rigid cuticle and flexible cuticle (or flexible cuticle structures). Flexible cuticle areas cover the segmental and joint regions, which allow insects to move or fly freely. The ability of flexible cuticle to maintain strength and

\footnotetext{
* Corresponding author. Hainan University, Haikou, Hainan 570228, China.

** Corresponding author.

E-mail addresses: qianhan@hainu.edu.cn (Q. Han), lij@vt.edu (J. Li).

1 Both authors contributed equally to this work.
}

stretch to a significant degree is believed being closely related to protein crosslinking. A highly flexible cuticle is particularly crucial in female mosquitoes because their abdomen must accommodate blood meal that can be equivalent to their own body weight.

Mosquitoes are disease vectors and cause more human suffering than any other organisms or insects (Le Coupanec et al., 2013; Mores et al., 2014; Diouf and Nour, 2017). Over one million people worldwide die from mosquito-borne diseases on yearly basis (Caraballo and King, 2014). These mosquitoes not only affect humans, but also have a potential to transmit several diseases to dogs, horses and other animals. The completion of genomes for several major disease vectors (i.e. Anopheles gambiae, Aedes aegypti and Culex quinquefasciatus) represents a major milestone towards developing a better overall understanding of mosquito physiology (Holt et al., 2002; Nene et al., 2007; Arensburger et al., 2010). These genome sequences revealed a number of insect specific gene families and even mosquito specific gene/protein families (such as cuticle-formation/hardening, blood feeding, blood digestion and ovary/egg development related gene families, etc.) that play essential physiological roles for mosquito survival. The absence of these gene families in other species suggests they could be ideal targets for mosquito control (Jasinskiene et al., 2003; Robich et al., 2007; Honnen et al., 2016). 


\section{Insect cuticular proteins}

As most insects undergo metamorphosis, they need to shred off their old cuticle and synthesize a new one to fit the body shape and size throughout their life cycles. The newly formed cuticle (Fig. 1), mainly composed of cuticular proteins, chitin, and sclerotizing reagents, needs to be hardened through the crosslinking between cuticular proteins and sclerotizing reagents. The ability to adapt to different environments leads to the success of insects in evolution and their widespread presence on earth likely is attributable to the protection of their cuticle. Cuticular proteins and chitin are major building blocks of insect cuticle. Identification of cuticular proteins and determination of their transcriptional profile are major steps forward for gaining a better understanding of cuticle formation and hardening, but exactly how individual cuticular proteins and chitin integrate to form highly protective cuticle structures remains to be fully established.

Cuticular protein crosslinking was thought to be involved in flexible cuticle formation (Tatarenkov et al., 2001; Vavricka et al., 2011). Cuticular proteins are either synthesized by the epidermal cells or transported from the hemolymph (Csikos et al., 1999). Arthropod cuticular proteins constitute a much diverse group (Andersen et al., 1995). Thirteen families of cuticular proteins have been reported in arthropods. They were named CPR (RR1, RR2, and RR3), CPF, CPFL, TWDL, CPAP1, CPAP3, CPLCA, CPLCG, CPLCW, CPLCP, and CPTC, respectively. Certain motifs or short stretches of amino acids in cuticular proteins are used for the identification of the proteins families. Some of the families are restricted to a particular insect order. The features of each family have been described previously (Willis, 2010; Willis et al., 2012). The majority of cuticular proteins belong to the family CPR, which has the Rebers and Riddiford Consensus sequence (R\&R Consensus; Pfam motif: PF00379) and was first identified by Rebers and Riddiford, (1988). Three subfamilies of CPR cuticular proteins have been reported. i.e. RR-1, RR-2, and RR-3 (Andersen, 1998, 2000; Karouzou et al., 2007). The classification was based on the presence of a Rebers and Riddiford (RR) consensus that was proposed to have affinity to chitin (Rebers et al., 1997). Cuticular proteins with RR-1 type domain are considered as flexible cuticular proteins and those with RR-2 type domain are predicted as rigid cuticular proteins (Andersen, 2000), which are used to classify flexible and rigid cuticular proteins in sequenced insect species in cuticle DB website (Magkrioti et al., 2004). However, there are exceptions. For example, RR-1 motif was also found to contribute to the differentiation of rigid cuticle during ecdysis in insects (Soares et al., 2007; Noh et al., 2015). RR-3 has only been identified in a few cuticular protein sequences and

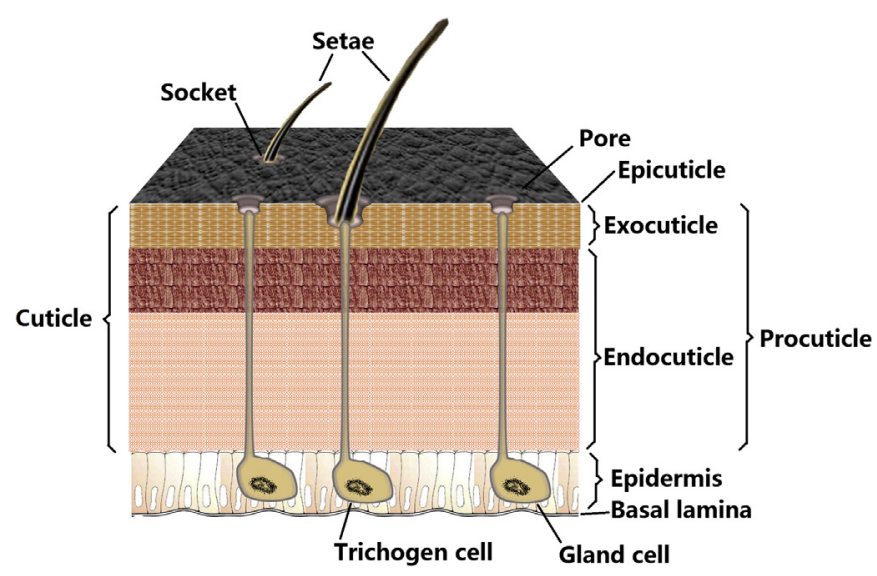

Fig. 1. Cuticle structure of insect (modified from Hackman and Goldberg, 1971). their role in cuticle formation remains to be established (Karouzou et al., 2007; Willis, 2010; Ioannidou et al., 2014). The $N$-terminal of the conserved motif of RR contains abundant hydrophilic amino acids, and the conservation of the sequence is related to the type of cuticle in which the epidermis is located. The consensus region of RR-2s, located in rigid cuticle, is conserved, but the consensus motif of RR-1s in flexible cuticle is variable in many species (Rebers and Riddiford, 1988; Willis, 2010). The conserved motif of RR has a binding site for chitin (Andersen et al., 1995) and its molecular conformation is $\beta$ sheet, which seems the necessary structural conformation for interacting with chitin. It has been proposed to cross link with reactive quinone species when binding with chitin (Guan et al., 2006; Arakane et al., 2012). Other small families also have characteristic motifs. The CPF family has no more than 44 conserved amino acid motif (CPF motif: PF 11018) (Togawa et al., 2007). CPFL (CPF-like) family lacks the conserved 44 amino acid residues but its $\mathrm{C}$-terminal regions are similar to those of the $\mathrm{CPF}$ family (Togawa et al., 2007). The CPAP1 and CPAP3 families have a peritrophin-A motif with six distinctly spaced cysteine residues (ChtBD2 domain: PF 01607); CPAP1 has one and CPAP2 has three ChtBD2 domains (Jasrapuria et al., 2012). The CPLCA (cuticular protein of low complexity A), CPLCG, CPLCW and CPLCP families do not possess the motifs described above for other cuticular protein families (He et al., 2007; Willis et al., 2012).

Whole genome sequencing of some insects helps identify cuticular proteins of the insects. Twenty eight cuticular proteins in CPR family have been reported in Apis mellifera (Consortium, 2006), 101 CPR cuticular protein genes reported in Drosophila melanogaster (Karouzou et al., 2007), 156 CPR genes identified in Anopheles gambiae (Cornman et al., 2008), 148 cuticular proteins in Bombyx mori (Futahashi et al., 2008), and 158 CPR cuticular protein genes identified and named in An. sinensis (Liu et al., 2017). Total cuticular proteins of A. mellifera, Dr. melanogaster, An. gambiae, B. mori and An. sinensis are 46,193, 257, 165, and 250, respectively (Liu et al., 2017). However, the exact number of cuticular protein varies from different research groups. For example, genome analysis suggested 238 putative cuticular proteins, including 136 CPR cuticular proteins in An. sinensis (Zhou et al., 2017), which is slightly different from what reported by another group (Liu et al., 2017).

Cuticular proteins play an important role in determining the diverse physical properties of cuticle by interactions among themselves and with chitin (Neville, 1993). The unique localization and cross-linking of specific cuticular proteins may be critical for the roles individual cuticular proteins plays. Flexibility or rigidity of cuticles is determined by the degree of cross-linking of some cuticular proteins together with dehydration. However, the functional significance and precise locations of cuticular proteins within a cuticle in insect are still not well established. Studies demonstrated that AgamCPF3 protein is localized in the adult exocuticle, while AgamCPLCG3/4 proteins are restricted to the endocuticle in An. gambiae (Vannini et al., 2014). CPR family RR-1s were localized in the procuticle of the soft intersegmental membrane except for one protein found in the endocuticle of rigid cuticle, while CPR family RR-2s were consistently found in rigid cuticle and not in flexible cuticle (Vannini and Willis, 2017). In Dr. melanogaster a single cuticular protein, Obstructor-E was reported being able to control the whole body shape of the fly (Tajiri et al., 2017). In the silk worm, Bombyx mori, mutation of the BmorCPR2 gene was described for being responsible for an abnormal distribution of internodes and intersegmental folds, leading to reductions in chitin content and decrease of tensile/stretch properties in larval cuticle (Qiao et al., 2014). Functional studies in the beetle Tribolium castaneum suggested the roles of four major cuticular proteins (TcCPR27, TCCPR18, TcCPR4 and TcCP30) in cuticle integrity and property and adult eclosion (Noh et al., 2016). 
In addition, several cuticular proteins are differentially expressed in insecticide resistant and susceptible strains in Culex pipiens pallens (Wang et al., 2015), Aedes aegypti (Pothikasikorn et al., 2010), An. gambiae (Nkya et al., 2014), An. stephensi (Vontas et al., 2007) and An. sinensis (Zhou et al., 2017). Experimental studies indicated that cuticular proteins could alter the sensitivity to deltamethrin in C. pipiens pallens (Fang et al., 2015). The possible involvement of some cuticular proteins in pesticide resistance may be through changing the cuticle property; thereby impeding the absorbance of insecticides.

\section{Insect cuticle pigmentation}

Cuticle pigmentation is a part of cuticle formation and hardening process. Numbers of chemical pigments, i.e., melanins, pterins, ommochromes, antraquinones, aphins, tertapyrroles, carotenoids and flavonoids/anthocyanins, are components of a variety of insect colors (Shamim et al., 2014). Among them, melanins, produced by tyrosine metabolism, are most extensively studied. Fig. 2 illustrates the commonly considered biochemical pathway involved in cuticle pigmentation and hardening in insects. This is often referred tyrosine-mediated cuticle pigmentation and sclerotization. Sclerotin is believed one of important cuticle components in insects, but the exact structure is poorly defined. $N$-acetyldopamine (NADA) and $N$ - $\beta$-alanyldopamine (NBAD) have been proposed the crosslinking precursors. It has been generally accepted that upon oxidation, their corresponding reactive quinones react with cuticular proteins, leading to the formation of insect sclerotins. Dopamine, derived from tyrosine, is the key component for the synthesis of both NADA and NBAD. Tyrosine is first hydroxylated to

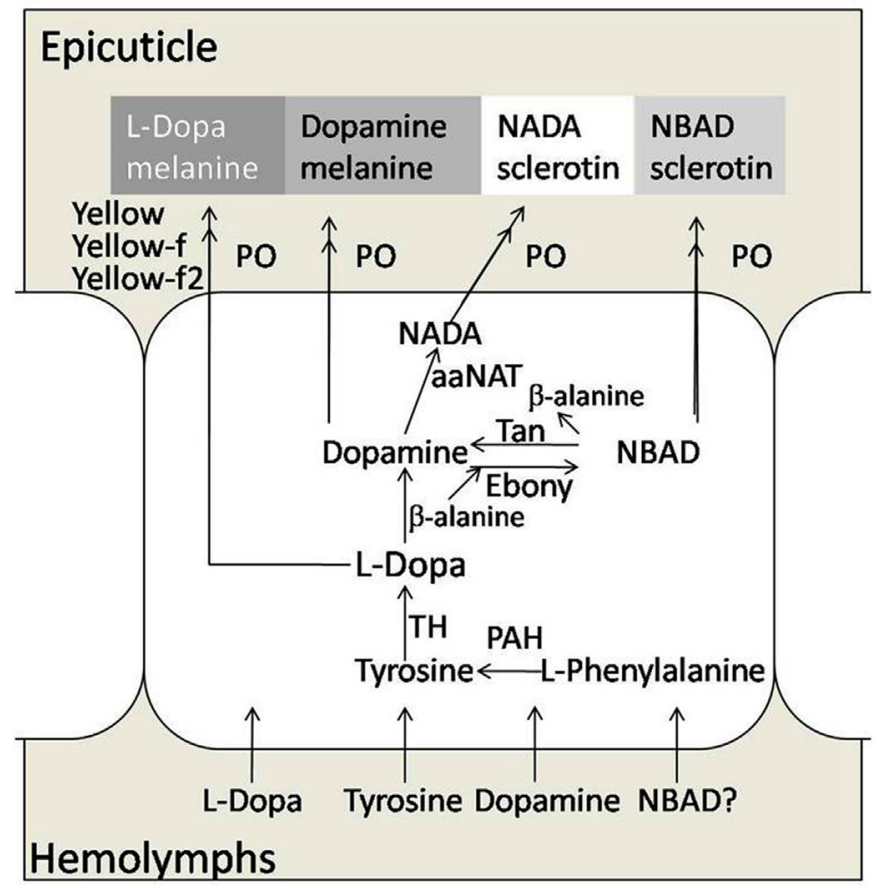

Fig. 2. Chemical pathway of cuticle pigmentation in Drosophila. The figure was modified from True at al. (2005). L-dopa as the precursor is catalyzed by different enzymes to constitute four different color rigid cuticles in Drosophila melanogaster. aaNAT, arylamine $\mathrm{N}$-acetyltransferase; DDC, dopa decarboxylase; Ebony, Ebony protein; NADA, $N$-acetyldopamine; NBAD, $N$ - $\beta$-alanyldopamine; PAH, phenylalanine hydroxylase; PO, phenoloxidase; Tan, Tan protein; TH, tyrosine hydroxylase. (For interpretation of the references to colour in this figure legend, the reader is referred to the web version of this article.) 3,4-dihydroxyphenylalanine (dopa) by a tyrosine hydroxylase $(\mathrm{TH})$ and the latter is then decarboxylated by dopa decarboxylase to dopamine (Andersen, 2010; Han et al., 2010; Vavricka et al., 2014). Dopamine can be either acetylated by arylalkylamine $\mathrm{N}$-acetyltransferase (aaNAT) to produce NADA (Mehere et al., 2011; Han et al., 2012) or conjugated with $\beta$-alanine to synthesize NBAD by $N$ - $\beta$-alanyl dopamine synthase (Ebony protein) in the presence of ATP (Hovemann et al., 1998). The tan gene encodes a hydroxylase, capable for hydrolyzing NBAD to dopamine and $\beta$-alanine (True et al., 2005) and the regulation of Ebony and tan protein is believed playing a key role in relating intensity of cuticle melanization and sclerotization (Wright, 1987). These catecholamine derivatives, which are synthesized in the epidermal cells and secreted into the cuticle, are the important elements of epidermal pigmentation and sclerotin. Hemolymphs can also produce the catecholamines as well as materials for the formation of cuticle (Wright, 1987). During cuticle hardening, phenoloxidase (PO) (in some species their laccases) oxidizes NADA and NBAD to their 0 -quinones that then react with nucleophilic groups in cuticular proteins (Andersen and Roepstorff, 1982; Vincent and Wegst, 2004; Suderman et al., 2006), leading to their crosslinking or cuticle coloration. Four different coloration pathways are believed being involved in Drosophila melanogaster. The Yellow proteins are required to produce black melanin. Among them, yellow-f and yellow $\mathrm{f} 2$ have been demonstrated being directly involved in melanization reaction by mediating conversion of dopachrome to 5,6-dihydroxyl indole (Walter et al., 1991; Han et al., 2002; Wittkopp et al., 2002), while ebony protein plays central role in the formation of tan pigments (Hovemann et al., 1998; Wittkopp et al., 2002). Dopamine melanin may be involved in the brownblack coloration. However, the detailed process leading to different coloration, including biochemical and molecular regulation of substrates and enzymes, remains to be substantiated.

Cuticle melanization has been studied in many insect species. $\mathrm{PO}$, dopachrome conversion enzyme (DCE) and dopa decarboxylase (DDC) are the key enzymes in cuticle melanization. Reactive quinones, derived from NADA and NBAD, are considered major cuticular protein crosslinking agents. NADA-quinone and/or NBADquinone-mediated cuticular protein crosslinking (or sclerotization) and cuticle melanization likely are primary biochemical processes involved in the formation of rigid cuticle in many species, but it is questionable if they equally apply to the formation of flexible cuticle.

\section{DDC and DOPAL synthase in insects}

Insect DDC, often termed aromatic amino acid decarboxylase (AAAD), is a pyridoxal phosphate dependent enzyme. This enzyme catalyzes decarboxylation of L-dopa and 5-hydroxyl-tryptophan (HTP) to dopamine and 5-hydroxytrptamine (5-HT), respectively in mammals (Sourkes, 1966). Many vertebrate, including mammals, indeed contains only a single DDC gene and the enzyme catalyzes the decarboxylation of both dopa and HTP, which likely explains why it was often named AAAD. Differing from vertebrate species, insects have several different DDC-like genes based on available sequenced insect genomes. For example, Drosophila melanogaster possesses five genes sharing high sequence identity with DDC. According to the latest gene annotations, Drosophila DDC-like genes include one typical DDC, two tyrosine decarboxylase-like proteins, and two $\alpha$-methyl dopa (AMD) resistant proteins. In mosquitoes, 6 to 7 DDC-like genes have been found for the high sequence identity (about 50\%) in this gene family. DDC in insects appears multifunctional, which contrasts to mammalian DDC. Insect DDC, like their mammalian counterparts, is responsible for the formation of some key neurotransmitters (Livingstone and Tempel, 1983; 
Osborne, 1996), but moreover, this insect DDC is also involved in other physiological processes, such as immune responses (Nappi et al., 1992; Davis et al., 2008), cuticle hardening (Huang et al., 2005; Paskewitz and Andreev, 2008) and egg-tanning (Ferdig et al., 1996).

It was indicated that Ae. aegypti homologue proteins of Drosophila AMD resistant proteins, encoded by AAEL010734 and AAEL010735 genes, are involved in the formation of protective, flexible cuticle in mosquitoes (Vavricka et al., 2011). These two genes had been named aromatic amino acid decarboxylase (AAAD) based on their high sequence identity (51\%) to mosquito DDC (AAEL014238). Although the predicted AAAD proteins use L-dopa as a substrate, they catalyze production of DOPAL instead of any aromatic amines. This highly reactive aldehyde derivative can directly participate in cuticular protein crosslinking. The Ae. aegypti proteins have been named DOPAL synthases and the transcriptional profile is closely correlated with cuticle development.

Early studies reported that some fruit flies are vulnerable (Sparrow and Wright, 1974) and some are resistant (Sherald and Wright, 1974) to AMD in their food supplies. Subsequently, the vulnerable or resistant phenotype was linked with a DDC-like gene, called $A M D$-resistant $(A M D-r)$ gene. It was later demonstrated that Drosophila AMD-resistant protein is resistant to the toxic compound, AMD (Black et al., 1987; Wang et al., 1996). Transcripts of the $A M D-r$ gene were detected in ovaries, in segment areas of the developing embryo and also in foregut, hindgut and the tracheal system. Noticeable defects in flexible cuticular structures have been observed in AMD-r gene mutants (Marsh and Wright, 1986; Wang and Marsh, 1995; Wang et al., 1996). Presence of AMD-r gene in many Drosophila species was subsequently discussed (Tatarenkov et al., 2001). Despite numerous efforts in trying to determine the specific activities/functions of Drosophila AMD resistant proteins, what exactly AMD resistant proteins do remained a mystery before the mosquito homologues were identified as DOPAL synthases (Vavricka et al., 2011). These previous studies have led to a general consensus that the AMD resistant gene is somehow related to the formation of un-melanized, flexible cuticle. Subsequent sequence comparative analysis and biochemical characterization of two Drosophila AMD resistant proteins demonstrated that both have the activity of DOPAL synthases (Tatarenkov et al., 2001; Vavricka et al., 2011).

It is intriguing that DDC and DOPAL synthase share high sequence identity, but mediate rather different chemical reactions. Decarboxylases are pyridoxal-5-phosphate (PLP) containing proteins. This PLP cofactor in DDC associates with its protein in a Schiffbase linkage formed through the aldehyde group of PLP and amino group of a conserved lysine residue (Lys302). The carboxyl group of D270 and the protonated pyridine nitrogen of PLP form a salt bridge, which is conserved in many PLP-containing enzymes. The PLP pyridine ring is stacked between residues A272 and H192. The phosphate moiety of PLP interacts with residues A148, S149 and N299 as well as with F103 from the other enzyme monomer of the dimer complex (Han et al., 2010). Although sequence comparison (Fig. 3) shows that Drosophila DDC and DOPAL synthase share similar cofactor binding residues, there is an apparent difference in chemical properties of PLP-binding residue between DDC and DOPAL. H192 and N192 occupy a similar position in the DDC and DOPAL synthase and the two residues seem to play dictating role in DDC and DOPAL-mediated reaction processes, respectively. Mutation of N192 or H192 in DOPAL synthase and H192 to N192 changed essentially the DDC mutant to a DAPOL synthase or a DOPAL synthase to DDC (Liang et al., 2017).

It has been commonly accepted that formation of quinonoid intermediate accounts for DDC-mediated reaction (Eliot and Kirsch, 2004). In PLP-mediated reactions, the possible chemical mechanisms, in general, are well-predicted and quite reasonable, but for enzyme mediated process, some subtle differences may lead major difference in their catalytic pathways. In DDC-mediated reaction, a unidentified residue functioning as a proton acceptor was proposed (Eliot and Kirsch, 2004) and our data suggest that H192 plays the key role for DDC-mediated process (Liang et al., 2017). The Asn192 in DOPAL synthase occupies essentially identical position as His192 in DDC, but inability for Asn192 in accepting or donating protein appears to impact largely the subsequent reaction of the reactive quinonoid. As a result, this intermediate reacts with oxygen, which is believed being driven by the instability of the quinonoid intermediate (Liang et al., 2017). Fig. 4 illustrates the proposed mechanisms for DDC- and DAPOL synthase-mediated reactions, respectively. These comparative studies complement the mechanism proposed for decarboxylase-catalyzed reaction and new insight toward comprehensive understanding of DOPAL synthase-mediated process.

A BLAST search according to the sequence identity and signature residues now suggests that DOPAL synthase is present in most insect species. Knockout studies in Aedes aegypti and Anopheles gambiae using their newly oviposited eggs showed that no embryo hatched in all injected experiment eggs (injected more 100 eggs in several experiments), while eggs injected with different control DNA displayed hatching rate at 50-60\% (the knockdown process leads to embryo injury, not all injected embryos are able to survival). It is disappointed for not being able to obtain observable phenotype, but inability to obtain phenotypic form through the procedure clearly suggests the importance of DOPAL synthase ( $\mathrm{Li}$ et al. unpublished data).

\section{Functions of DOPAL synthase in insects}

As described above, L-dopa is the key precursor for the cuticle tanning and melanization pathway in the formation of rigid cuticle, but L-dopa is also the immediate precursor for the production of DOPAL predicted to crosslink cuticular proteins in flexible cuticle (Vavricka et al., 2010, 2014). DOPAL, produced by DOPAL synthase, is highly reactive, and can directly participate in protein crosslinking through its interaction with free amino groups on cuticular proteins (such as the free amine from lysine residues). This might be somewhat comparable with the stabilization process of elastin and collagen in humans and many other species. Newly formed elastin and collagen fibers are fragile, but cross-linked fibers have very high tensile strength. During their maturation, some lysine and hydroxyl-lysine residues in elastin and collagen are oxidized to aldehyde derivatives by lysine oxidase (Narayanan et al., 1978; Miao et al., 2005; Kumashiro et al., 2006). While there are several lysine oxidases in human and many other species, there is no single similar enzyme is present in insects after extensive search. For mosquitoes and many other insects, the ability to achieve rapid formation of protective cuticle is vital (due in part to their relatively short life-span). Because sclerotization and pigmentation reactions lead to the formation of rigid, pigmented cuticle, these reactions likely are avoided in the flexible cuticle hardening process. Production of DOPAL from dopa by DOPAL synthase, apparently, is much more rapid than the oxidation of lysine residues to aldehyde derivatives in large protein molecules by lysine oxidase.

Based on the high reactivity of DOPAL, the defects of flexible cuticle structures in Drosophila DOPAL synthase gene mutants, and the preferential transcription of DOPAL synthase in mosquito abdomen where flexible cuticle is dominant, it is reasonable to propose that DOPAL-mediated cuticular protein crosslinking is a novel, efficient strategy utilized by mosquitoes to produce protective, yet highly flexible cuticle in a timely manner. In DOPAL synthase-catalyzed reactions, oxygen is converted to hydrogen 
$\begin{array}{ccccccc} & 80 & 90 & 100 & 110 & 120 & 130 \\ \text { DOPAL synthase WQSPHMHAYYPTSTSYPS I V GEMLA S FF GV I GF SW ICSPACTELEVVVMDWLAKFLKLPAHFQHASDGPG } \\ \text { DDC }\end{array}$

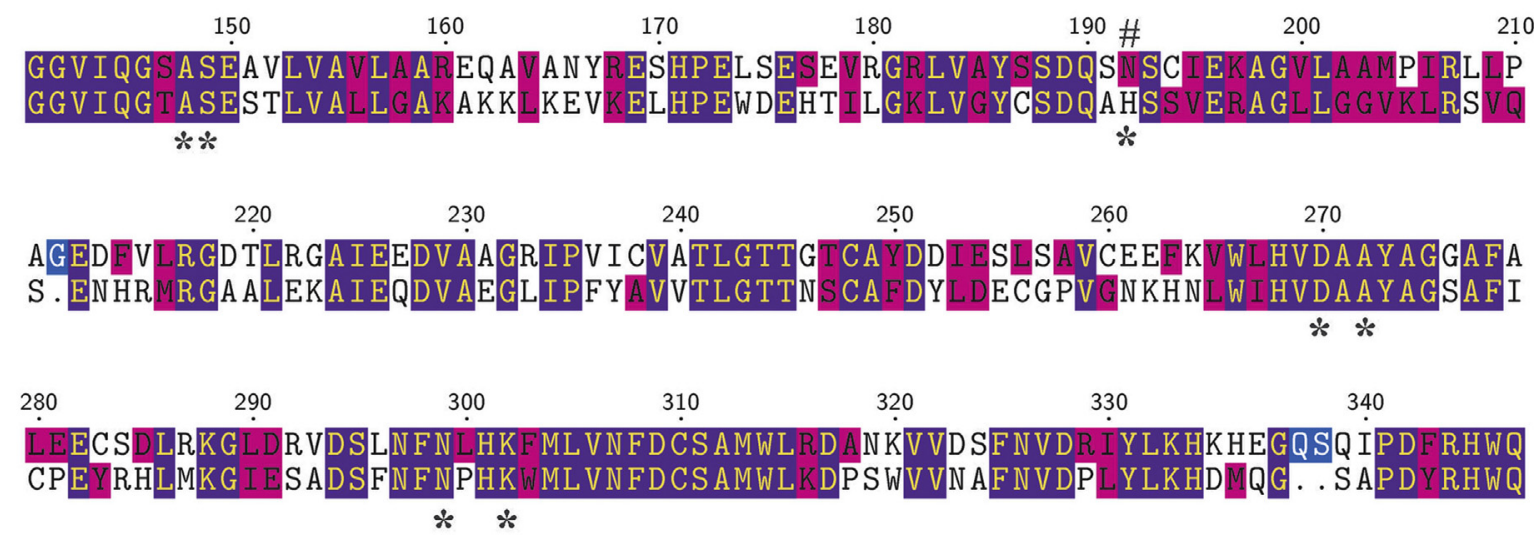

Fig. 3. Sequence comparison of Drosophila DDC (NP724164) and DOPAL synthase/amd resistant protein A (NP_476592). Letters with blue and red background are fully conserved and strongly conserved amino acid residues, respectively. Residues interacting with PLP are labeled with "**, different residues binding PLP between two enzymes is labeled with "\#”. K303 in DOPAL synthase and K302 in DDC are involved in the formation of the internal aldimine with PLP. The ruler for residue numbers are for Drosophila DDC. (For interpretation of the references to colour in this figure legend, the reader is referred to the web version of this article.)

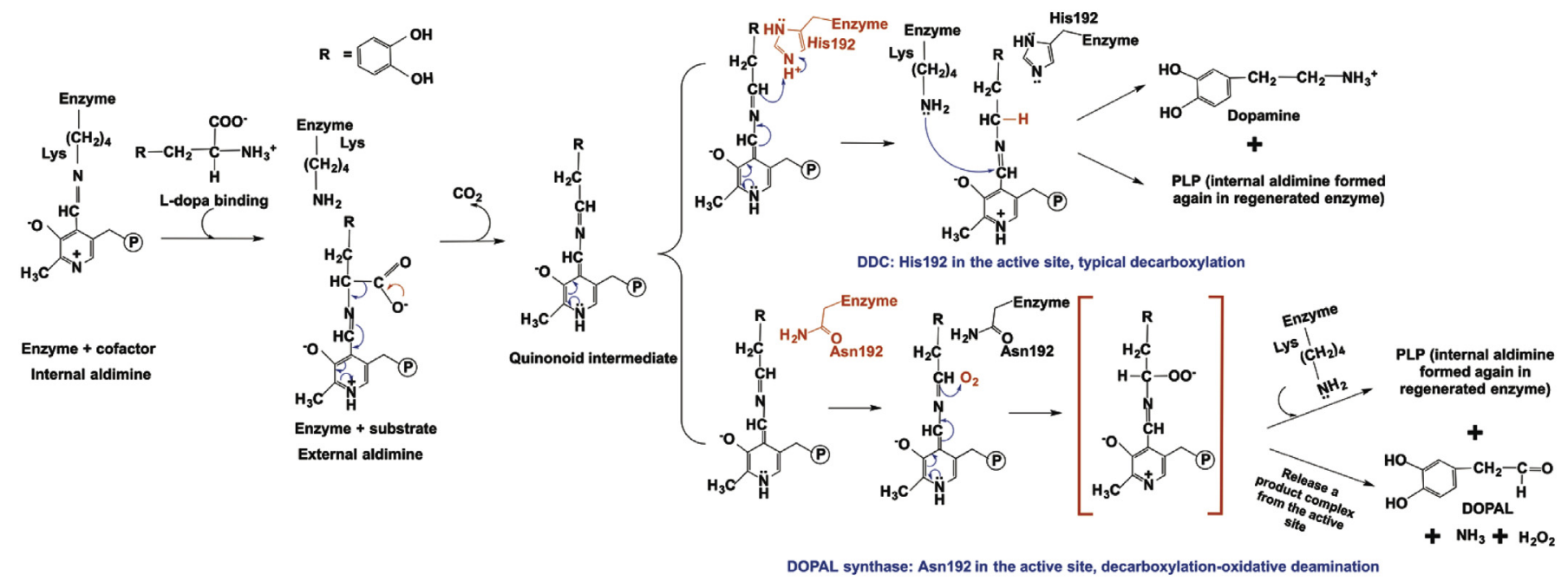

Fig. 4. The proposed mechanistic role of His192 and Ans192 in typical decarboxylation and decarboxylation-oxidative deamination catalyzed by DDC and DOPAL synthase respectively (Liang et al., 2017).
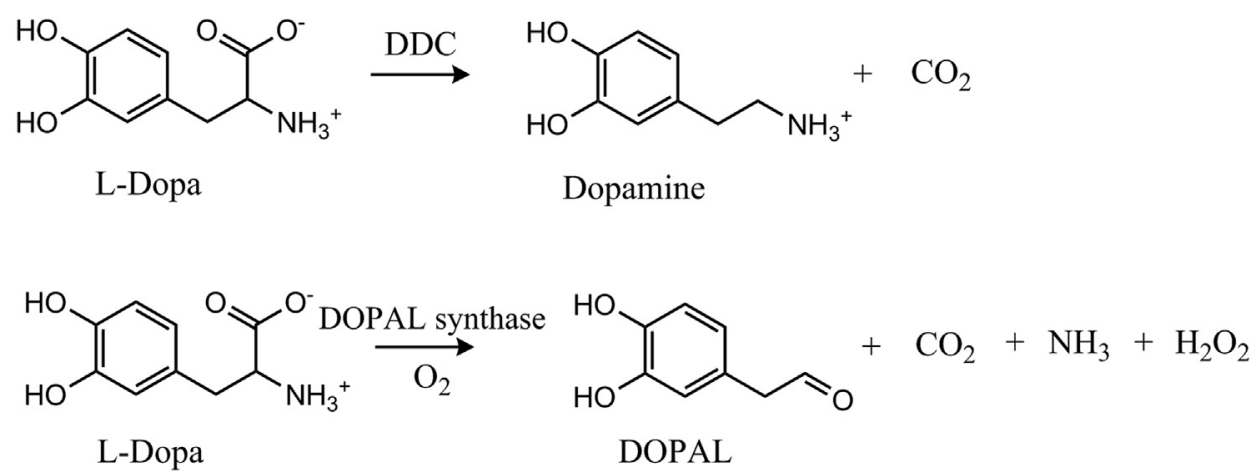

Fig. 5. The chemical reactions catalyzed by DDC and DOPAL synthase. 
peroxide $\left(\mathrm{H}_{2} \mathrm{O}_{2}\right)$ during the oxidative deamination process (Fig. 5). Under normal circumstances, both DOPAL and $\mathrm{H}_{2} \mathrm{O}_{2}$ are toxic compounds. While $\mathrm{H}_{2} \mathrm{O}_{2}$ can be inactivated by catalase, DOPAL would be highly toxic due to its ability to inactivate and aggregate proteins if allowed to circulate. Within regions of flexible cuticle production, however, $\mathrm{H}_{2} \mathrm{O}_{2}$ might promote further oxidation of the DOPAL catechol ring, leading to further cuticular protein crosslinking. Therefore, this highly toxic pathway of dopa to DOPAL may play a vital role in the production of protective flexible cuticle through mediating protein crosslinking.

It is interesting to discover DOPAL synthase because there is no MAO in insects and cannot produce DOPAL directly from dopamine (Vavricka et al., 2014). DOPAL synthase mediates a complicated, but some details steps in catalysis remain to be elucidated. Evolvement of DOPAL synthase in insects apparently was an adaption of insects to better survival in their living environments. The data regarding this insect enzyme and its possible mechanisms should provide the momentum for a comprehensive understanding of its role in cuticle formation.

\section{Conclusion and perspectives}

New functions could be evolved from an existing protein or a similar protein, which is driven essentially by environmental forces during adaptation. Defect of unpigmented, flexible cuticle constructions in Drosophila amd-r gene mutants, suggests a clear requirement for the functions of this protein in cuticle formation. Presence of DOPAL synthases might somewhat decrease cuticle sclerotization by NADA and NBAD and melanization by channeling L-dopa to DOPAL; thereby increasing the flexibility of articular structures. Although there have been a number of studies concern DOPAL synthase, many aspects concerning the enzyme and its catalytic reaction remain to be clarified, especially the specific interaction of DOPAL with the major cuticular proteins and the relationship of the DOPAL synthase and other cuticle hardening enzymes (TH, PO, DDC, etc.) involved in the formation of rigid cuticle. More specifically, since the flexible cuticle of mosquito is associated with the physiological, ethological and immunological functions, the mechanisms for preventing flexible cuticle to form rigid ones should be particularly interesting. With the advent of new biochemical and molecular biological tools, the enzyme function in cuticle formation and insect immune responses should be clear established in the near future.

\section{Acknowledgements}

This work was supported by the National Natural Science Foundation of China (31472186), the Natural Science Foundation of Hainan Province (20163048) and Hainan University Research Fund (hdkytg201702).

\section{References}

Andersen, S.O., 1998. Amino acid sequence studies on endocuticular proteins from the desert locust, Schistocerca gregaria. Insect Biochem. Mol. Biol. 28, 421-434.

Andersen, S.O., 2000. Studies on proteins in post-ecdysial nymphal cuticle of locust, Locusta migratoria, and cockroach, Blaberus craniifer. Insect Biochem. Mol. Biol. 30, 569-577.

Andersen, S.O., 2010. Insect cuticular sclerotization: a review. Insect Biochem. Mol. Biol. 40, 166-178.

Andersen, S.O., Roepstorff, P., 1982. Sclerotization of insect cuticle: III. An unsaturated derivative of $\mathrm{N}$-acetyldopamine and its role in sclerotization. Insect Biochem. Mol. Biol. 12, 8.

Andersen, S.O., Hojrup, P., Roepstorff, P., 1995. Insect cuticular proteins. Insect Biochem. Mol. Biol. 25, 153-176.

Arakane, Y., Lomakin, J., Gehrke, S.H., Hiromasa, Y., Tomich, J.M., Muthukrishnan, S. Beeman, R.W., Kramer, K.J., Kanost, M.R., 2012. Formation of rigid, non-flight forewings (elytra) of a beetle requires two major cuticular proteins. PLoS
Genet. 8, e1002682.

Arensburger, P. Megy, K., Waterhouse, R.M., Abrudan, J., Amedeo, P., Antelo, B., Bartholomay, L., Bidwell, S., Caler, E., Camara, F., et al., 2010. Sequencing of Culex quinquefasciatus establishes a platform for mosquito comparative genomics. Science 330, 86-88.

Black, B.C., Pentz, E.S., Wright, T.R., 1987. The alpha methyl dopa hypersensitive gene, 1(2)amd, and two adjacent genes in Drosophila melanogaster: physical location and direct effects of amd on catecholamine metabolism. Mol. Gen. Genet. 209, 306-312.

Caraballo, H., King, K., 2014. Emergency department management of mosquitoborne illness: malaria, dengue, and West Nile virus. Emerg. Med. Pract. 16 (1-23) quiz 23-24.

Consortium THGS, 2006. Insights into social insects from the genome of the honeybee Apis mellifera. Nature 443, 931-949.

Cornman, R.S., Togawa, T., Dunn, W.A., He, N., Emmons, A.C., Willis, J.H., 2008. Annotation and analysis of a large cuticular protein family with the R\&R Consensus in Anopheles gambiae. BMC Genomics 9, 22

Csikos, G., Molnar, K., Borhegyi, N.H., Talian, G.C., Sass, M., 1999. Insect cuticle, an in vivo model of protein trafficking. J. Cell Sci. 112 (Pt 13), 2113-2124.

Davis, M.M., Primrose, D.A., Hodgetts, R.B., 2008. A member of the p38 mitogenactivated protein kinase family is responsible for transcriptional induction of Dopa decarboxylase in the epidermis of Drosophila melanogaster during the innate immune response. Mol. Cell Biol. 28, 4883-4895.

Diouf, K., Nour, N.M., 2017. Mosquito-borne diseases as a global health problem: implications for pregnancy and travel. Obstet. Gynecol. Surv. 72, 309-318.

Eliot, A.C., Kirsch, J.F., 2004. Pyridoxal phosphate enzymes: mechanistic, structural, and evolutionary considerations. Annu. Rev. Biochem. 73, 383-415.

Fang, F., Wang, W., Zhang, D., Lv, Y., Zhou, D., Ma, L., Shen, B., Sun, Y., Zhu, C., 2015. The cuticle proteins: a putative role for deltamethrin resistance in Culex pipiens pallens. Parasitol. Res. 114, 4421-4429.

Ferdig, M.T., Li, J., Severson, D.W., Christensen, B.M., 1996. Mosquito dopa decarboxylase cDNA characterization and blood-meal-induced ovarian expression. Insect Mol. Biol. 5, 119-126.

Futahashi, R., Okamoto, S. Kawasaki, H., Zhong, YS, Iwanaga, M., Mita, K, Fujiwara, H., 2008. Genome-wide identification of cuticular protein genes in the silkworm. Bombyx Mori. Insect Biochem. Mol. Biol. 38, 1138-1146.

Gibbs, A., Mousseau, T.A., Crowe, J.H., 1991. Genetic and acclimatory variation in biophysical properties of insect cuticle lipids. Proc. Natl. Acad. Sci. U. S. A. 88, 7257-7260.

Guan, X., Middlebrooks, B.W., Alexander, S., Wasserman, S.A., 2006. Mutation of TweedleD, a member of an unconventional cuticle protein family, alters body shape in Drosophila. Proc. Natl. Acad. Sci. U. S. A. 103, 16794-16799.

Hackman, R.H., Goldberg, M., 1971. Studies on the hardening and darkening of insect cuticles. J. Insect. Physiol. 17, 335-347.

Han, Q., Fang, J., Ding, H., Johnson, J.K., Christensen, B.M., Li, J., 2002. Identification of Drosophila melanogaster yellow-f and yellow- $\mathrm{f} 2$ proteins as dopachromeconversion enzymes. Biochem. J. 368, 333-340.

Han, Q., Ding, H., Robinson, H., Christensen, B.M., Li, J., 2010. Crystal structure and substrate specificity of Drosophila 3,4-dihydroxyphenylalanine decarboxylase. PLoS One 5, e8826.

Han, Q., Robinson, H., Ding, H., Christensen, B.M., Li, J., 2012. Evolution of insect arylalkylamine $\mathrm{N}$-acetyltransferases: structural evidence from the yellow fever mosquito, Aedes aegypti. Proc. Natl. Acad. Sci. U. S. A. 109, 11669-11674.

He, N., Botelho, J.M., McNall, R.J., Belozerov, V., Dunn, W.A., Mize, T., Orlando, R., Willis, J.H., 2007. Proteomic analysis of cast cuticles from Anopheles gambiae by tandem mass spectrometry. Insect Biochem. Mol. Biol. 37, 135-146.

Holt, R.A., Subramanian, G.M., Halpern, A., Sutton, G.G., Charlab, R., Nusskern, D.R., Wincker, P., Clark, A.G., Ribeiro, J.M., Wides, R., et al., 2002. The genome sequence of the malaria mosquito Anopheles gambiae. Science 298, 129-149.

Honnen, A.C., Johnston, P.R., Monaghan, M.T., 2016. Sex-specific gene expression in the mosquito Culex pipiens f. molestus in response to artificial light at night. BMC Genomics 17, 22.

Hovemann, B.T., Ryseck, R.P., Walldorf, U., Stortkuhl, K.F., Dietzel, I.D., Dessen, E., 1998. The Drosophila ebony gene is closely related to microbial peptide synthetases and shows specific cuticle and nervous system expression. Gene 221, $1-9$.

Huang, C.Y., Chou, S.Y., Bartholomay, L.C., Christensen, B.M., Chen, C.C., 2005. The use of gene silencing to study the role of dopa decarboxylase in mosquito melanization reactions. Insect Mol. Biol. 14, 237-244.

Ioannidou, Z.S., Theodoropoulou, M.C., Papandreou, N.C., Willis, J.H., Hamodrakas, S.J., 2014. CutProtFam-Pred: detection and classification of putative structural cuticular proteins from sequence alone, based on profile hidden Markov models. Insect Biochem. Mol. Biol. 52, 51-59.

Jasinskiene, N., Coates, C.J., Ashikyan, A., James, A.A., 2003. High efficiency, sitespecific excision of a marker gene by the phage P1 cre-loxP system in the yellow fever mosquito, Aedes aegypti. Nucleic Acids Res. 31, e147.

Jasrapuria, S., Specht, C.A., Kramer, K.J., Beeman, R.W., Muthukrishnan, S., 2012. Gene families of cuticular proteins analogous to peritrophins (CPAPs) in Tribolium castaneum have diverse functions. PLoS One 7, e49844.

Karouzou, M.V., Spyropoulos, Y., Iconomidou, V.A., Cornman, R.S., Hamodrakas, S.J., Willis, J.H., 2007. Drosophila cuticular proteins with the R\&R Consensus: annotation and classification with a new tool for discriminating RR-1 and RR-2 sequences. Insect Biochem. Mol. Biol. 37, 754-760.

Kumashiro, K.K., Ho, J.P., Niemczura, W.P., Keeley, F.W., 2006. Cooperativity between the hydrophobic and cross-linking domains of elastin. J. Biol. Chem. 281, 
23757-23765

Le Coupanec, A., Babin, D., Fiette, L., Jouvion, G., Ave, P., Misse, D., Bouloy, M., Choumet, V., 2013. Aedes mosquito saliva modulates Rift Valley fever virus pathogenicity. PLoS Negl. Trop. Dis. 7, e2237.

Liang, J., Ding, H., Han, Q., Li, J., 2017. Biochemical identification of residues that discriminate between 3,4-dihydroxyphenylalanine decarboxylase and 3,4dihydroxyphenylacetaldehyde synthase-mediated reactions. Insect biochem. Mol. Biol. (91), 34-43.

Liu, B.Q., Qiao, L., He, O.Y., Zhou, Y., Ren, S., Chen, B., 2017. Genome-wide identification, characterization and evolution of cuticular protein genes in the malaria vector Anopheles sinensis (Diptera: Culicidae). Insect Sci. https://doi.org/10.1111/ 1744-7917.12483.

Livingstone, M.S., Tempel, B.L., 1983. Genetic dissection of monoamine neurotransmitter synthesis in Drosophila. Nature 303, 67-70.

Magkrioti, C.K., Spyropoulos, I.C., Iconomidou, V.A., Willis, J.H., Hamodrakas, S.J., 2004. cuticleDB: a relational database of Arthropod cuticular proteins. BMC Bioinforma. 5, 138.

Marsh, J.L., Wright, T.R., 1986. Evidence for regulatory variants of the dopa decarboxylase and alpha-methyldopa hypersensitive loci in Drosophila. Genetics 112, 249-265.

Mehere, P., Han, Q., Christensen, B.M., Li, J., 2011. Identification and characterization of two arylalkylamine $\mathrm{N}$-acetyltransferases in the yellow fever mosquito, Aedes aegypti. Insect Biochem. Mol. Biol. 41, 707-714.

Miao, M., Cirulis, J.T., Lee, S., Keeley, F.W., 2005. Structural determinants of crosslinking and hydrophobic domains for self-assembly of elastin-like polypeptides. Biochemistry 44, 14367-14375.

Mores, C.N., Christofferson, R.C., Davidson, S.A., 2014. The role of the mosquito in a dengue human infection model. J. Infect. Dis. 209 (Suppl. 2), S71-S78.

Moussian, B., 2010. Recent advances in understanding mechanisms of insect cuticle differentiation. Insect Biochem. Mol. Biol. 40, 363-375.

Moussian, B., Seifarth, C., Muller, U., Berger, J., Schwarz, H., 2006. Cuticle differentiation during Drosophila embryogenesis. Arthropod Struct. Dev. 35, 137-152.

Nappi, A.J., Carton, Y., Vass, E., 1992. Reduced cellular immune competence of a temperature-sensitive dopa decarboxylase mutant strain of Drosophila melanogaster against the parasite Leptopilina boulardi. Comp. Biochem. Physiol. B 101, 453-460.

Narayanan, A.S., Page, R.C., Kuzan, F., Cooper, C.G., 1978. Elastin cross-linking in vitro. Studies on factors influencing the formation of desmosines by lysyl oxidase action on tropoelastin. Biochem. J. 173, 857-862.

Nene, V., Wortman, J.R., Lawson, D., Haas, B., Kodira, C., Tu, Z.J., Loftus, B., Xi, Z., Megy, K., Grabherr, M., et al., 2007. Genome sequence of Aedes aegypti, a major arbovirus vector. Science 316, 1718-1723.

Neville, A.C., 1993. Biology of Fibrous Composites : Development beyond the Cell Membrane/A.C. Neville. Cambridge University Press, New York, NY, USA.

Nkya, T.E., Poupardin, R., Laporte, F., Akhouayri, I., Mosha, F., Magesa, S., Kisinza, W. David, J.P., 2014. Impact of agriculture on the selection of insecticide resistance in the malaria vector Anopheles gambiae: a multigenerational study in controlled conditions. Parasit. Vectors 7, 480.

Noh, M.Y., Muthukrishnan, S., Kramer, K.J., Arakane, Y., 2015. Tribolium castaneum RR-1 cuticular protein TcCPR4 is required for formation of pore canals in rigid cuticle. PLoS Genet. 11, e1004963.

Noh, M.Y., Muthukrishnan, S., Kramer, K.J., Arakane, Y., 2016. Cuticle formation and pigmentation in beetles. Curr. Opin. Insect Sci. 17, 1-9.

Ortiz-Urquiza, A., Keyhani, N.O., 2013. Action on the surface: entomopathogenic fungi versus the insect cuticle. Insects 4, 357-374.

Osborne, R.H., 1996. Insect neurotransmission: neurotransmitters and their receptors. Pharmacol. Ther. 69, 117-142.

Paskewitz, S.M., Andreev, O., 2008. Silencing the genes for dopa decarboxylase or dopachrome conversion enzyme reduces melanization of foreign targets in Anopheles gambiae. Comp. Biochem. Physiol. B Biochem. Mol. Biol. 150, 403-408.

Pesch, Y.Y., Riedel, D., Behr, M., 2017. Drosophila Chitinase 2 is expressed in chitin producing organs for cuticle formation. Arthropod Struct. Dev. 46, 4-12.

Pothikasikorn, J., Boonplueang, R., Suebsaeng, C., Khaengraeng, R., Chareonviriyaphap, T., 2010. Feeding response of Aedes aegypti and Anopheles dirus (Diptera: Culicidae) using out-of-date human blood in a membrane feeding apparatus. J. Vector Ecol. 35, 149-155.

Qiao, L., Xiong, G., Wang, R.X., He, S.Z., Chen, J., Tong, X.L., Hu, H., Li, C.L., Gai, T.T. Xin, Y.Q, et al., 2014. Mutation of a cuticular protein, BmorCPR2, alters larval body shape and adaptability in silkworm. Bombyx Mori. Genet. 196, 1103-1115.

Rebers, J.E., Riddiford, L.M., 1988. Structure and expression of a Manduca sexta larval cuticle gene homologous to Drosophila cuticle genes. J. Mol. Biol. 203, 411-423.

Rebers, J.E., Niu, J., Riddiford, L.M., 1997. Structure and spatial expression of the Manduca sexta MSCP14.6 cuticle gene. Insect Biochem. Mol. Biol. 27, 229-240.

Robich, R.M., Rinehart, J.P., Kitchen, L.J., Denlinger, D.L., 2007. Diapause-specific gene expression in the northern house mosquito, Culex pipiens $L$., identified by suppressive subtractive hybridization. J. Insect Physiol. 53, 235-245.

Shamim, G., Ranjan, S.K., Pandey, D.M., Ramani, R., 2014. Biochemistry and biosynthesis of insect pigments. Eur. J. Entomology 111, 149-164.

Sherald, A.F. Wright, T.R., 1974. The analog inhibitor, alpha-methyl dopa, as a screening agent for mutants elevating levels of dopa decarboxylase activity in Drosophila melanogaster. Molec Gen. Genet. 25-36.

Shibata, T., Ariki, S., Shinzawa, N., Miyaji, R., Suyama, H., Sako, M., Inomata, N., Koshiba, T., Kanuka, H., Kawabata, S., 2010. Protein crosslinking by transglutaminase controls cuticle morphogenesis in Drosophila. PLoS One 5, e13477.

Soares, M.P., Elias-Neto, M., Simoes, Z.L., Bitondi, M.M., 2007. A cuticle protein gene in the honeybee: expression during development and in relation to the ecdysteroid titer. Insect Biochem. Mol. Biol. 37, 1272-1282.

Sourkes, T.L., 1966. Dopa decarboxylase: substrates, coenzyme, inhibitors. Pharmacol. Rev. 18, 53-60.

Sparrow, J.C., Wright, T.R., 1974. The selection for mutants in Drosophila melanogaster hypersensitive to alpha-methyl dopa, a dopa decarboxylase inhibitor: Molec Gen. Genet. 127-141.

Suderman, R.J., Andersen, S.O., Hopkins, T.L., Kanost, M.R., Kramer, K.J., 2003. Characterization and cDNA cloning of three major proteins from pharate pupal cuticle of Manduca sexta. Insect Biochem. Mol. Biol. 33, 331-343.

Suderman, R.J., Dittmer, N.T., Kanost, M.R., Kramer, K.J., 2006. Model reactions for insect cuticle sclerotization: cross-linking of recombinant cuticular proteins upon their laccase-catalyzed oxidative conjugation with catechols. Insect Biochem. Mol. Biol. 36, 353-365.

Tajiri, R., Ogawa, N., Fujiwara, H., Kojima, T., 2017. Mechanical control of whole body shape by a single cuticular protein obstructor-e in Drosophila melanogaster. PLoS Genet. 13, e1006548.

Tatarenkov, A., Zurovcova, M., Ayala, F.J., 2001. Ddc and amd sequences resolve phylogenetic relationships of Drosophila. Mol. Phylogenet Evol. 20, 321-325.

Togawa, T., Augustine Dunn, W., Emmons, A.C., Willis, J.H., 2007. CPF and CPFL, two related gene families encoding cuticular proteins of Anopheles gambiae and other insects. Insect Biochem. Mol. Biol. 37, 675-688.

True, J.R., Yeh, S.D., Hovemann, B.T., Kemme, T., Meinertzhagen, I.A., Edwards, T.N., Liou, S.R., Han, Q., Li, J., 2005. Drosophila tan encodes a novel hydrolase required in pigmentation and vision. PLoS Genet. 1, e63.

Vannini, L. Willis, J.H., 2017. Localization of RR-1 and RR-2 cuticular proteins within the cuticle of Anopheles gambiae. Arthropod Struct. Dev. 46, 13-29.

Vannini, L., Reed, T.W., Willis, J.H., 2014. Temporal and spatial expression of cuticular proteins of Anopheles gambiae implicated in insecticide resistance or differentiation of M/S incipient species. Parasit. Vectors 7, 24.

Vavricka, C.J., Christensen, B.M., Li, J., 2010. Melanization in living organisms: a perspective of species evolution. Protein Cell 1, 830-841.

Vavricka, C., Han, O. Huang, Y., Erickson, S.M., Harich, K., Christensen, B.M., Li, J. 2011. From L-dopa to dihydroxyphenylacetaldehyde: a toxic biochemical pathway plays a vital physiological function in insects. PLoS One 6, e16124.

Vavricka, C.J., Han, Q., Mehere, P., Ding, H., Christensen, B.M., Li, J., 2014. Tyrosine metabolic enzymes from insects and mammals: a comparative perspective. Insect Sci. 21, 13-19.

Vincent, J.F., Wegst, U.G., 2004. Design and mechanical properties of insect cuticle Arthropod Struct. Dev, 33, 187-199.

Vontas, J., David, J.P., Nikou, D., Hemingway, J., Christophides, G.K., Louis, C. Ranson, H., 2007. Transcriptional analysis of insecticide resistance in Anopheles stephensi using cross-species microarray hybridization. Insect Mol. Biol. 16, 315-324.

Walter, M.F., Black, B.C., Afshar, G., Kermabon, A.Y., Wright, T.R., Biessmann, H., 1991 Temporal and spatial expression of the yellow gene in correlation with cuticle formation and dopa decarboxylase activity in Drosophila development. Dev. Biol. 147, 32-45.

Wang, D., Marsh, J.L., 1995. Developmental regulation of the alpha-methyldopa hypersensitive gene of Drosophila melanogaster. Dev. Biol. 168, 598-612.

Wang, D., Marsh, J.L., Ayala, F.J., 1996. Evolutionary changes in the expression pattern of a developmentally essential gene in three Drosophila species. Proc. Natl. Acad. Sci. U. S. A. 93, 7103-7107.

Wang, W. Lv, Y., Fang, F. Hong, S., Guo, O., Hu, S., Zou, F., Shi, L., Lei, Z., Ma, K., et al., 2015. Identification of proteins associated with pyrethroid resistance by iTRAQbased quantitative proteomic analysis in Culex pipiens pallens. Parasites Vectors $8,95$.

Willis, J.H., 2010. Structural cuticular proteins from arthropods: annotation, nomenclature, and sequence characteristics in the genomics era. Insect Biochem. Mol. Biol. 40, 189-204.

Willis, J.H., Papandreou, N.C., Iconomidou, V.A., Hamodrakas, S.J., 2012. Cuticular proteins. Insect Mol. Biol. Biochem. 134-166.

Wittkopp, P.J., True, J.R., Carroll, S.B., 2002. Reciprocal functions of the Drosophila yellow and ebony proteins in the development and evolution of pigment patterns. Development 129, 1849-1858.

Wright, T.R., 1987. The genetics of biogenic amine metabolism, sclerotization, and melanization in Drosophila melanogaster. Adv. Genet. 24, 127-222.

Zhou, D., Duan, B., Sun, Y., Ma, L., Zhu, C., Shen, B., 2017. Preliminary characterization of putative structural cuticular proteins in the malaria vector Anopheles sinensis. Pest Manag. Sci. https://doi.org/10.1002/ps.4649. 